\title{
Anthracene Fluorescence Quenching by a Tetrakis (Ketocarboxamide) Cavitand
}

\author{
Tibor Zoltan Janosi, ${ }^{1,2}$ Jouko Korppi-Tommola, ${ }^{3}$ Zsolt Csok, ${ }^{4,5}$ Laszlo Kollar, ${ }^{4,5}$ \\ Pasi Myllyperkio, ${ }^{3}$ and Janos Erostyak ${ }^{1,2}$ \\ ${ }^{1}$ Institute of Physics, University of Pecs, Hungary \\ ${ }^{2}$ Szentagothai Research Centre, Spectroscopy Research Group, University of Pecs, Ifjúságút 6, H-7624 Pécs, Hungary \\ ${ }^{3}$ Nanoscience Center, University of Jyvaskyla, Finland \\ ${ }^{4}$ Department of Inorganic Chemistry, University of Pecs, Hungary \\ ${ }^{5}$ Pécs Research Group for Selective Syntheses, Hungarian Academy of Sciences, Hungary
}

Correspondence should be addressed to Tibor Zoltan Janosi; tzjanosi@gamma.ttk.pte.hu

Received 12 June 2014; Accepted 8 July 2014; Published 6 August 2014

Academic Editor: Renata Diniz

Copyright ( 2014 Tibor Zoltan Janosi et al. This is an open access article distributed under the Creative Commons Attribution License, which permits unrestricted use, distribution, and reproduction in any medium, provided the original work is properly cited.

Quenching of both fluorescence lifetime and fluorescence intensity of anthracene was investigated in the presence of a newly derived tetrakis (ketocarboxamide) cavitand at various concentrations. Time-correlated single photon counting method was applied for the lifetime measurements. A clear correlation between the fluorescence lifetime of anthracene as a function of cavitand concentration in dimethylformamide solution was observed. The bimolecular collisional quenching constant was derived from the decrease of lifetime. Fluorescence intensity was measured in the emission wavelength region around $400 \mathrm{~nm}$ as a result of excitation at $280 \mathrm{~nm}$. Effective quenching was observed in the presence of the cavitand. The obtained Stern-Volmer plot displayed upward curvature. The results did not follow even extended Stern-Volmer behavior, often used to describe deviations from static bimolecular quenching. To explain our results we adopted the Smoluchowski model and obtained a reasonable estimate for the molecular radius of the cavitand in solution.

\section{Introduction}

Cavitands [1] are a class of molecules that contain a conformationally rigid cavity. As a result of their shape, the cavitands and the related bowl-shaped molecules have been used as hosts in host-guest complexes. They have become increasingly important in supramolecular chemistry and nanochemistry due to their potential in applications such as sensors, nanoreactors, and drug delivery systems [2-6]. As the donor-acceptor pair is formed, the charge distributions of the donor and the acceptor are modified and are different from those of the isolated molecules. Fluorescence spectroscopy - both static and time resolved - may be used to study such modifications and even bring evidence of donoracceptor pair formation.

In the present study, the interaction between a tetrakis (ketocarboxamide) cavitand (1) (Figure 1) and anthracene was investigated in order to test the ability of (1) to transport molecules selectively.

(1) was synthesized according to the procedure we have described before [7]. In short, using piperidine and the corresponding tetraiodocavitand under high-pressure $(90$ bar CO pressure) palladium-catalyzed aminocarbonylation conditions resulted in excellent chemoselectivities towards this tetrasubstituted, "double-carbonylated" compound. The selective and highly variable functionalization of the basic deepened cavitand skeleton is a prerequisite for any further applications including molecular recognition and selective transport processes [8].

It was estimated that the relatively small circumference of anthracene would allow it to penetrate into the host cavity. Fluorescence properties of anthracene in several solvents [911] have been studied hence forming a solid background for observations of noncollisional-that is, due to complex 
formation-quenching behavior in solutions where guest and the host were present at varying relative concentrations. Since there are several molecular interactions such as vibronic coupling, energy transfer, and conformational changes that may serve as a source of fluorescence quenching, care was taken in analyzing the quenching results with two different models, using the classical Stern-Volmer plots as well as the Smoluchowski-type quenching model [12].

\section{Material and Methods}

The tetrakis (ketocarboxamide) cavitand (1) was synthesized as previously described [7]. Its molecular structure is presented in Figure 1.

Both anthracene and $\mathbf{1}$ were dissolved in spectroscopic grade $N, N$-dimethylformamide (DMF) delivered by SigmaAldrich (product number: 154814).

The concentration of anthracene solution was kept constant at $1 \mathrm{mM}$. The concentrations of 1 were $10,20,40,60,80$, 100,150 , and $200 \mu \mathrm{M}$.

The ultraviolet and visible absorption were measured in a $1 \mathrm{~mm}$ quartz cuvette. A fluorescence spectrometer (PerkinElmer LS 55) with xenon lamp was used to obtain fluorescence spectra. Time-correlated single photon counting (TCSPC) method was used to obtain the fluorescence lifetimes of the solutions. TCSPC measurements were performed with a commercial PicoQuant HydraHarp 400 TCSPC data acquisition system. PicoQuant PLS290 laser source with $\sim 900$ ps pulse duration served as an excitation source. The lifetimes were estimated using least squares fitting technique with one-exponential decay model.

\section{Results and Discussion}

3.1. Absorption. Figure 2 shows the measured absorption spectra of pure 1 (dotted line, $c=200 \mu \mathrm{M}$ ) and pure anthracene (solid line, $c=1 \mathrm{mM}$ ). The dashed lines on Figure 2 represent the absorption of the solution of anthracene and cavitand in various concentrations.

It turned out that the total absorption of the sample was a linear combination of the absorbances of the two constituents. This means that, in solution, the two molecules do have only a weak interaction with each other or even no interaction. In complexation one would expect visible shifts in the spectra.

3.2. Fluorescence Lifetime. Figure 3 shows the fluorescence lifetimes of anthracene in the mixtures as a function of the concentration of the cavitand quencher (1). The excitation wavelength was $290 \mathrm{~nm}$, while the emission was detected at $404 \mathrm{~nm}$. There is a remarkable and nearly linear decrease in the lifetime as the function of the concentration of $\mathbf{1}$. It is worth noting that the scale of $y$-axis starts from 3.70 , so the change-even at the highest concentration-is less than $4 \%$.

Shorter fluorescence lifetimes were observed in the presence of 1 that indicates some kind of dynamic quenching effect between the anthracene and 1. In order to gain more information from this data, we created the Stern-Volmer plot (Figure 4).
3.3. Collisional Quenching. When an additional constituent is added into a solution of a fluorescent species normally fluorescence quenching occurs. This is seen as reduction of fluorescence intensity and shortening of fluorescence lifetime. One of known quenching mechanisms is collisional quenching that not only reduces the spectral intensity of fluorescence but also shortens the lifetime.

Collisional quenching of the fluorescence lifetime is described by the Stern-Volmer equation [13]:

$$
\frac{\tau_{0}}{\tau}-1=k_{C} \tau_{0} Q=K_{C} Q,
$$

where $Q$ is the quencher's concentration, $\tau_{0}$ and $\tau$ are, respectively, the fluorescence lifetimes in the absence and in the presence of quencher, and $k_{C}$ is the bimolecular quenching constant.

The bimolecular quenching constant reflects the accessibility of the quencher to the excited state molecules.

Plotting $\left(\tau_{0} / \tau-1\right)$ as a function of the concentration yields a linear plot with a slope equal to $K_{C}$. The linear fitting on the data of the present study results in the following constants: $K_{C}$ is $208 \pm 9 \mathrm{M}^{-1}$ while the bimolecular quenching constant is $k_{C}=5.37 \pm 0.23 \cdot 10^{10} \mathrm{M}^{-1} \mathrm{~s}^{-1}$.

Pure collisional quenching of anthracene in different solvents by various quenchers has been previously reported $[14,15]$.

The value of $K_{C}$ was earlier measured [14] in the concentration range of 10 and $30 \mathrm{M}^{-1}$ in n-heptane, n-hexane, and $\mathrm{CCl}_{4}$ in the presence of fullerene. In another work [15] $K_{C}$ was obtained in the range of $2-106 \mathrm{M}^{-1}$. Here the solvents were toluene, methanol, and diethylene glycol, while triethylamine and 4-butylaniline were used as quencher.

Collisional frequency $(f)$ of a quencher with the fluorophore may be described by the following equation:

$$
f=k_{0} \cdot Q \text {, }
$$

where $k_{0}$ is the diffusion-controlled bimolecular rate constant, $\left[k_{0}\right]=\mathrm{M}^{-1} \mathrm{~s}^{-1}$.

This constant can be obtained from the Smoluchowski equation $[16,17]$ that describes the diffusion of molecules that are larger than the solvent molecules. This is the case for the anthracene and $\mathbf{1}$ when dissolved in DMF. Consider

$$
k_{0}=4 \pi N_{A} R D
$$

where $N_{A}$ is the Avogadro number $\left(N_{A}=6 \cdot 10^{23} \mathrm{~mol}^{-1}\right), R$ is the collision radius, and $D$ denotes the sum of the diffusion coefficients of cavitand $\mathbf{1}\left(D_{C}\right)$ and anthracene $\left(D_{A}\right)$. It is usually assumed that the collision radius is equal to the sum of molecular radii [18] of the anthracene $\left(R_{A}\right)$ and $\mathbf{1}\left(R_{C}\right)$.

We get

$$
k_{0}=4 \pi N_{A}\left(R_{A}+R_{C}\right)\left(D_{A}+D_{C}\right) .
$$

Diffusion coefficients can be calculated by using the Stokes-Einstein equation [19]:

$$
D=\frac{k T}{6 \pi \eta R},
$$




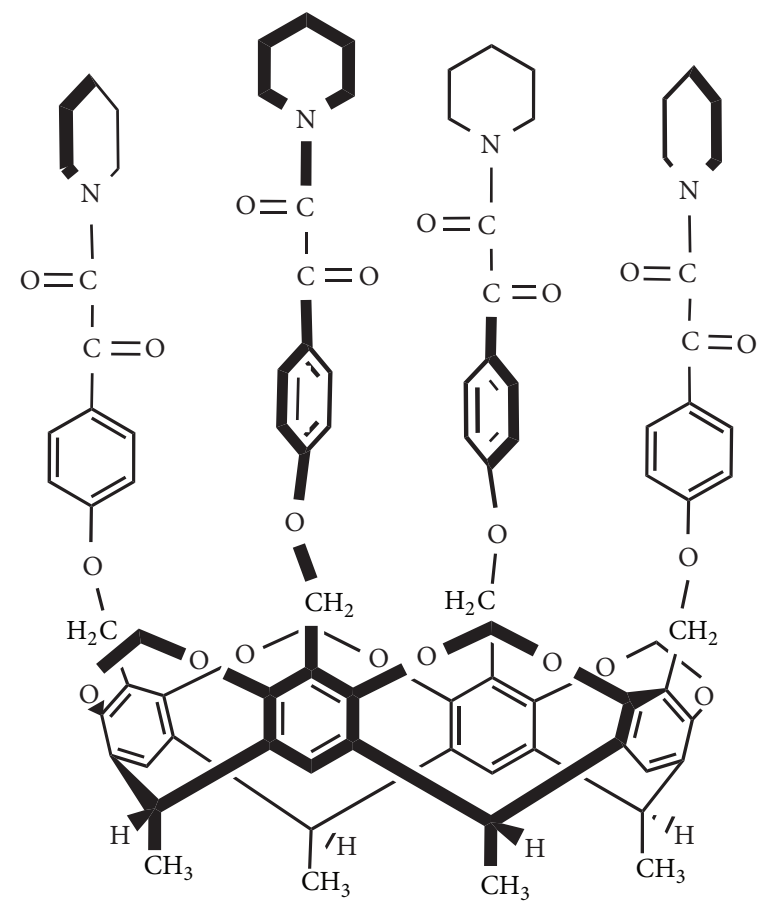

FIGURE 1: Structure of $\mathbf{1}$.

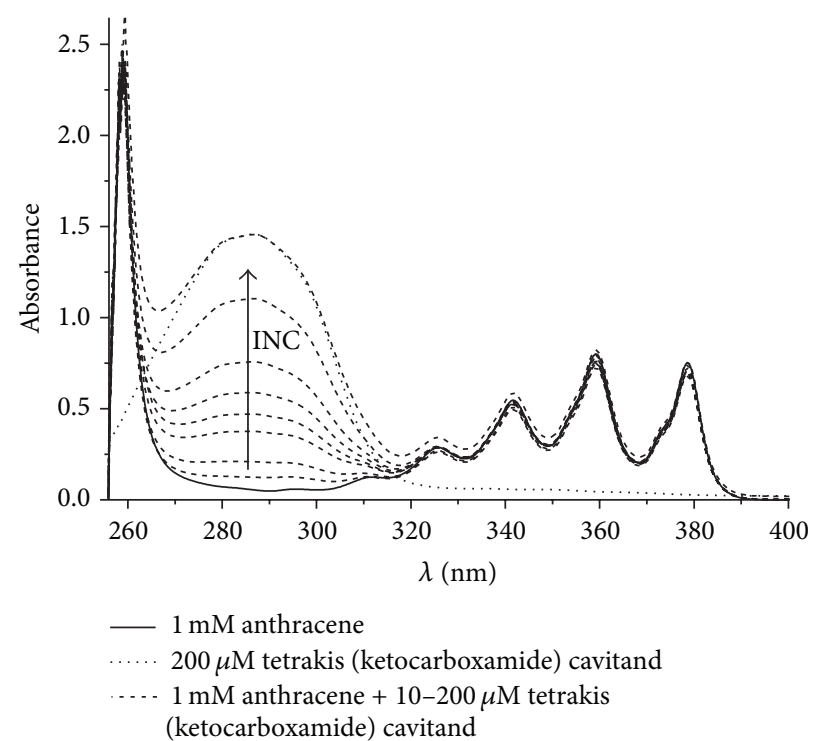

Figure 2: Absorption spectrum of anthracene $(1 \mathrm{mM}), \mathbf{1}(200 \mu \mathrm{M})$ and anthracene in the presence of different concentrations of 1 (the arrow indicates increasing concentration of $\mathbf{1}$ ).

where $k=1.38 \cdot 10^{-23} \mathrm{~J} / \mathrm{K}$ is the Boltzmann's constant, $R$ is the radius of the molecule, $\eta=0.92 \mathrm{~Pa} \cdot \mathrm{s}$ is the viscosity of the DMF, and $T=293 \mathrm{~K}$ is the absolute temperature.

With these constants we get the following equation for $k_{0}$ :

$$
k_{0}=1.758 \cdot 10^{9} \mathrm{M}^{-1} \mathrm{~s}^{-1}\left(2+\frac{R_{A}}{R_{C}}+\frac{R_{C}}{R_{A}}\right) .
$$

The diameter of the anthracene molecule may be approximated by assuming it to have the same volume as its van der
Walls volume $R_{A}=0.4 \mathrm{~nm}$ [20]. From a molecular modeling calculation we estimate the radius of the cavitand molecule as $3 \mathrm{~nm}$.

With the parameters presented above the estimated value of the diffusion-controlled bimolecular rate constant becomes $1.5 \cdot 10^{10} \mathrm{M}^{-1} \mathrm{~s}^{-1}$.

The measured bimolecular constant $\left(5.37 \cdot 10^{10} \mathrm{M}^{-1} \mathrm{~s}^{-1}\right)$ is of the same order of magnitude as the theoretically estimated value, but clearly higher. Since the experimental rate constant 


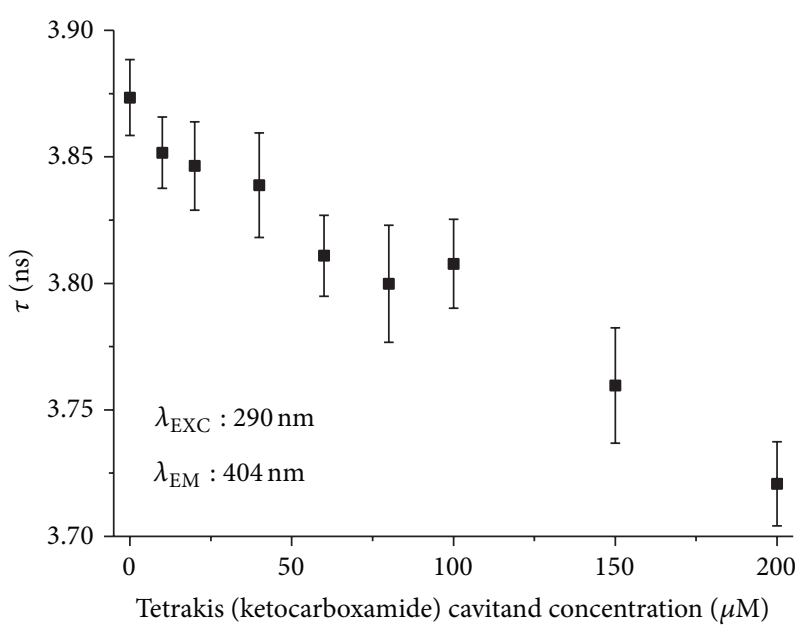

FIGURE 3: Anthracene fluorescence lifetime in the presence of different concentrations of $\mathbf{1}$.

is much higher than the constant estimated from diffusion properties, some additional quenching mechanisms must be present [21].

3.4. Fluorescence Intensity. Also the intensity dependence of the anthracene fluorescence in presence of $\mathbf{1}$ was studied. The measured fluorescence intensities were corrected in order to eliminate the effect of apparent quenching, which is the decline of excitation intensity due the absorption of $\mathbf{1}$ at the excitation wavelength. This effect was compensated by the following consideration.

The sample is characterized by the concentration $(c)$ and the molar extinction coefficient $(\varepsilon)$ in a cuvette with an optical path length of $d$. The initial intensity of the light is $I_{0}$.

According to the Beer-Lambert law, the intensity of light as a function of optical path $(x)$ is

$$
I_{x}=I_{0} 10^{-\varepsilon \cdot c \cdot x} .
$$

We get the effective excitation intensity $\left(F_{\text {eff }}\right)$ by calculating the integral of the intensity along the optical path length:

$$
\begin{aligned}
F_{\text {eff }} & =\int_{0}^{d} I_{0} 10^{-\varepsilon \cdot c \cdot x} d x \\
& =\frac{-I_{0}}{\varepsilon \cdot c \cdot \ln 10}\left[10^{-\varepsilon \cdot c \cdot x}\right]_{0}^{d} \\
& =\frac{-I_{0}}{\varepsilon \cdot c \cdot \ln 10}\left(10^{-\varepsilon \cdot c \cdot d}-10^{-\varepsilon \cdot c \cdot 0}\right) \\
& =F_{\text {eff }}=\frac{-I_{0}}{\varepsilon \cdot c \cdot \ln 10}\left(10^{-\varepsilon \cdot c \cdot d}-1\right) \\
F_{\text {eff }} & =I_{0} \frac{1-10^{-\varepsilon \cdot c \cdot d}}{\varepsilon \cdot c \cdot \ln 10} .
\end{aligned}
$$

The theoretical excitation intensity for a totally transparent solution would be

$$
F_{t}=I_{0} \cdot d
$$

The fluorescence excitation intensity correction factor is defined as

$$
f=\frac{F_{t}}{F_{\mathrm{eff}}}=\frac{\varepsilon \cdot c \cdot d \cdot \ln 10}{1-10^{-\varepsilon \cdot c \cdot d}} .
$$

For more details, see $[22,23]$.

In the present study reference fluorescence intensity $\left(I_{0}\right)$ was first measured without the quencher and then at different quencher concentrations. The corrected emission spectra of anthracene at two excitation wavelengths $(280 \mathrm{~nm}$ and $360 \mathrm{~nm}$ ) have been plotted in Figure 5. There was no observable change in the fluorescence intensity when the anthracene was excited at $360 \mathrm{~nm}$ (upper-right corner of Figure 5). On the main graph, it can be seen that the emission induced by $280 \mathrm{~nm}$ light was strongly diminished in the presence of 1 . The shape of the spectra was not altered by the quenching; that is, the intensity decrease was wavelengthindependent.

To create the Stern-Volmer plot, the measured $I$ and $I_{0}$ values at the peak intensity at $402 \mathrm{~nm}$ were used.

If we assume that the fluorophore is quenched both by the collision and by the complex formation with the same quencher, the modified Stern-Volmer equation [12] describes the fractional fluorescence remaining $\left(I / I_{0}\right)$. Consider

$$
\frac{I_{0}}{I}=\left(1+K_{C} Q\right)\left(1+K_{S} Q\right),
$$

where $Q$ is the quencher's concentration and $K_{C}$ and $K_{S}$ are the collisional and static quenching constants, respectively.

Rearranging (11) we get

$$
\frac{\left(I_{0} / I-1\right)}{Q}=\left(K_{C}+K_{S}\right)+\left(K_{C} \cdot K_{S}\right) Q .
$$

As it was mentioned earlier, the collisional quenching appears in both the fluorescence lifetime and the fluorescence intensity and causes a same decrease in both of them. Combining (1) and (11) we can separate the contribution of the static part from the total quenching:

$$
\frac{I_{0}}{I}=\frac{\tau_{0}}{\tau}\left(1+K_{S} Q\right) .
$$

Figure 6 shows the Stern-Volmer plots for the quenching of the anthracene fluorescence intensity in the presence of 1. The open symbols indicate the total quenching, that is, $I_{0} / I-1$, while the filled symbols represent the quenching due to complex formation according to $(13),\left(I_{0} / I\right)\left(\tau / \tau_{0}\right)-1$.

A positive deviation from the linearity can be observed; the plot remains concave toward the $Y$-axis. This upward curvature of the apparent quenching shows that (11) is not applicable for the analysis of this quenching. Furthermore, if we plot the $\left(I_{0} / I-1\right) / Q$ as the function of cavitand's concentration, the resulted curve is not a straight line as it should be according to (12). These facts prove that modified Stern-Volmer equation (11) does not describe the observed quenching process properly. 


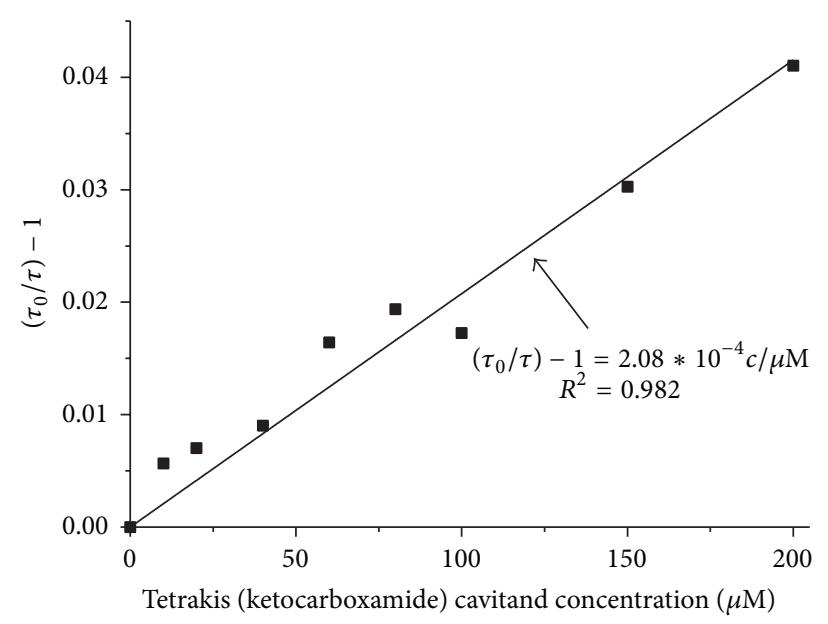

FIGURE 4: Stern-Volmer plot for the fluorescence lifetime of anthracene.

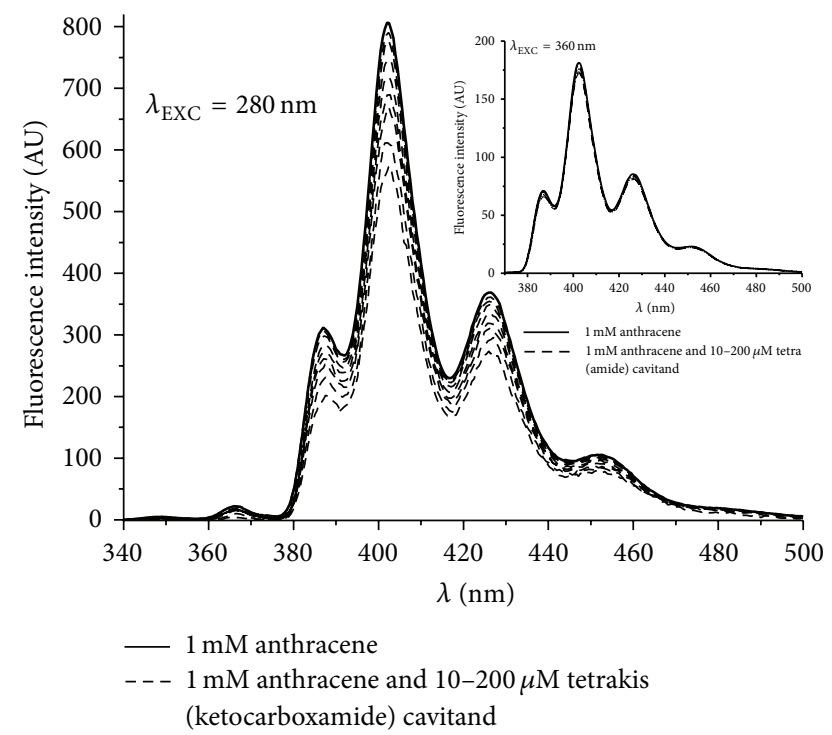

FIGURE 5: Corrected fluorescence spectra of anthracene in the absence and in the presence of different concentrations (10-200 $\mu \mathrm{M})$ of the cavitand quencher (1). The excitation wavelength was $\lambda_{\mathrm{EXC}}=$ $280 \mathrm{~nm}$ for the main and $\lambda_{\text {EXC }}=360 \mathrm{~nm}$ in the graph on the upperright corner.

3.5. Sphere of Action Quenching. For further analysis, we used the sphere of action quenching model [24]. This model is based on simple and intuitive assumption on the molecular level of the process. The instantaneous quenching takes place if the quencher and the fluorophore are in contact or very close to each other at the moment when the fluorescent molecule happens to be excited. In these encounters only a fraction of the excited fluorophore molecules is quenched by the collision. We assume random distribution of the quencher and the fluorophore at the moment of excitation. If the solutions are diluted, then we can use Poisson distribution to describe the probability of the quencher and the fluorophore molecules located close enough to each other for the quenching to occur. It is important to highlight that RET is not involved in the quenching processes, as there is no remarkable overlap between the fluorescence spectra of quenched and the absorption spectra of quencher.

If $W$ is the fraction of the excited state molecules quenched by some dynamic effect, then

$$
\frac{I_{0}}{I}=\frac{1+K_{\mathrm{SV}} Q}{W}
$$

where $Q$ is the quencher's concentration and $K_{\mathrm{SV}}$ is the SternVolmer quenching constants.

The probability of $n$ quencher molecules being located in volume $V$ is precisely described by the binomial distribution. If the number of quencher molecules is sufficiently large and the average number of quencher molecules in the investigated volume is low enough, then the Poisson distribution is a good approximation of the binomial distribution.

According to the Poisson distribution, the probability of $n$ quencher molecules located in volume $V$ is

$$
P(n, V)=\frac{\lambda_{V}^{n}}{n !} e^{-\lambda_{V}}
$$

where $\lambda_{V}$ is the average number of quenchers in volume $V$ :

$$
\lambda_{V}=Q \cdot V \cdot N_{A} .
$$

We use the Poisson distribution since the present case represents a low $\lambda_{V}$ value. $\lambda_{V}$ is often expressed in percent; for example, in our case $\lambda_{V}=4 \%$ would mean that there is one cavitand molecule in volume $V$ surrounded by 25 anthracene molecules.

The probability that there is no quencher near a fluorophore is

$$
P(0, V)=e^{-\lambda_{V}}
$$

This probability is equal to the fraction $W$ of the excited fluorophore molecules that are quenched by collisions. Consider

$$
W=e^{-\lambda_{V}}
$$




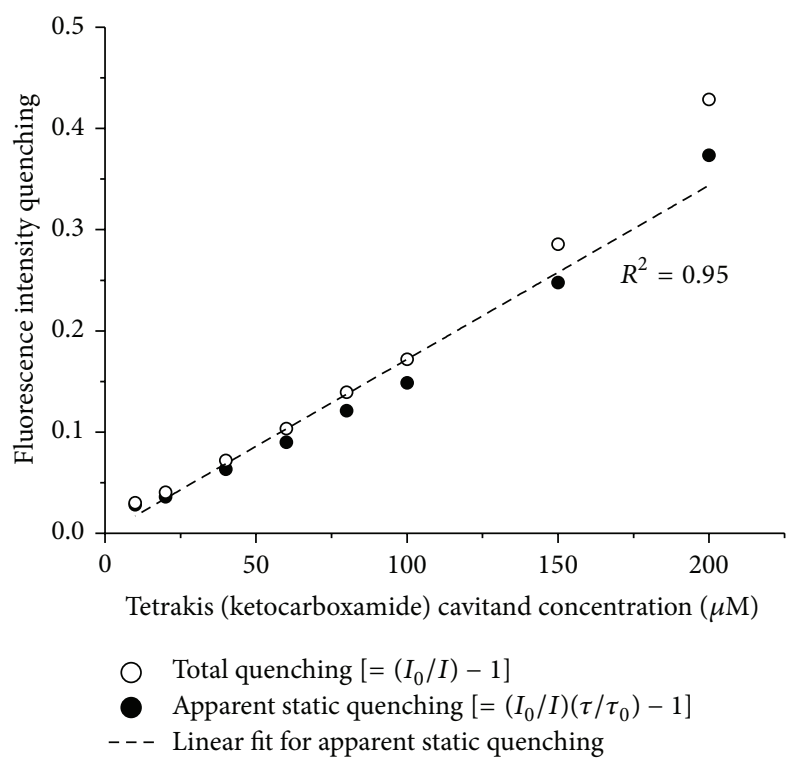

FIGURE 6: Stern-Volmer plot for the total and for the apparent static quenching of anthracene by 1.

From (14) and (18), we get

$$
\frac{I_{0}}{I}=\left(1+K_{\mathrm{SV}} \mathrm{Q}\right) e^{\mathrm{Q} \cdot V \cdot N_{A}}
$$

If $\lambda_{V}$ is small, that is, $Q$ and $V$ are small enough, we can use linear approximation:

$$
W=e^{-\lambda_{V}} \approx 1-\lambda_{V}
$$

Now (14) may be rewritten [25]:

$$
\frac{\left(1-I / I_{0}\right)}{\mathrm{Q}}=K_{\mathrm{SV}} \frac{I}{I_{0}}+\frac{1-W}{\mathrm{Q}}=K_{\mathrm{SV}} \frac{I}{I_{0}}+V \cdot N_{A} .
$$

Thus $V$ can be derived from the analysis of the $(1-$ $\left.\left(I / I_{0}\right)\right) / Q$ plot (Figure 6) as the function of $I / I_{0}$, and it represents the active volume surrounding the quencher.

The action radius can be calculated from the active volume $(V)$ as follows:

$$
V=\frac{4}{3} r^{3} \pi
$$

The fitting (Figure 7) resulted in $258 \pm 68 \mathrm{M}^{-1}$ for the value of $V \cdot N_{A}$; thus the calculated active volume is $43 \pm 11.3 \cdot 10^{-23} \mathrm{l}$, while the estimated action radius is $4.68 \pm 0.4 \mathrm{~nm}$. This value is slightly higher than the sum of diameter of anthracene and $\mathbf{1}$ and is likely the distance limit for the instantaneous quenching.

It was previously mentioned that the use of Poisson distribution is limited by the value of $\lambda_{V}$. It is usually recommended to use Poisson distribution below the $\lambda_{V}$ value of $8 \%$.

In our case, the value of $\lambda_{V}$ at the highest quencher concentration is $5 \%$. This is its maximum because $\lambda_{V}$ is changing linearly with the quencher's concentration. It means
TABLE 1: Comparison of the constants derived from the sphere of action quenching model $\left(K_{\mathrm{SV}}\right.$ is the Stern-Volmer constant and $R$ is the action radius) $[10,11]$.

\begin{tabular}{lcc}
\hline Quencher & $K_{\mathrm{SV}}\left(\mathrm{M}^{-1}\right)$ & $R(\mathrm{~nm})$ \\
\hline Aniline & $12-38$ & $1.2-3.8$ \\
Allyl 2,4-dinitrophenyl ether & $766-1410$ & $0.61-0.72$ \\
$\mathbf{1}$ & $1782 \pm 78$ & $4.68 \pm 0.4$ \\
\hline
\end{tabular}

that our system fits this restriction, and the use of Poisson distribution is appropriate.

The $R^{2}$ of fitting is 0.986 , which means that the applied sphere of action quenching model describes the observed change in fluorescence intensity properly.

Positive deviation from the Stern-Volmer plot at the anthracene quenching has been previously observed [9-11].

In two of these studies, aniline and allyl 2,4-dinitrophenyl ether induced the anthracene fluorescence quenching in various solvents, and the effects were described by the sphere of action quenching model. The calculated constants are compared in Table 1.

The higher value of the action radius $(4.68 \pm 0.4 \mathrm{~nm})$ is in accordance with the bigger molecule size. The relatively high Stern-Volmer constant indicates that $\mathbf{1}$ is able to effectively quench the anthracene fluorescence.

\section{Conclusions}

In summary, it has been shown that a newly derived tetrakis (ketocarboxamide) cavitand causes simultaneous dynamic and prompt quenching of anthracene fluorescence. The dynamic quenching was explained by the collision mechanism, while the Smoluchowski model of sphere of action quenching was applied to describe the contribution of the prompt effect. The derived constants are in good agreement 


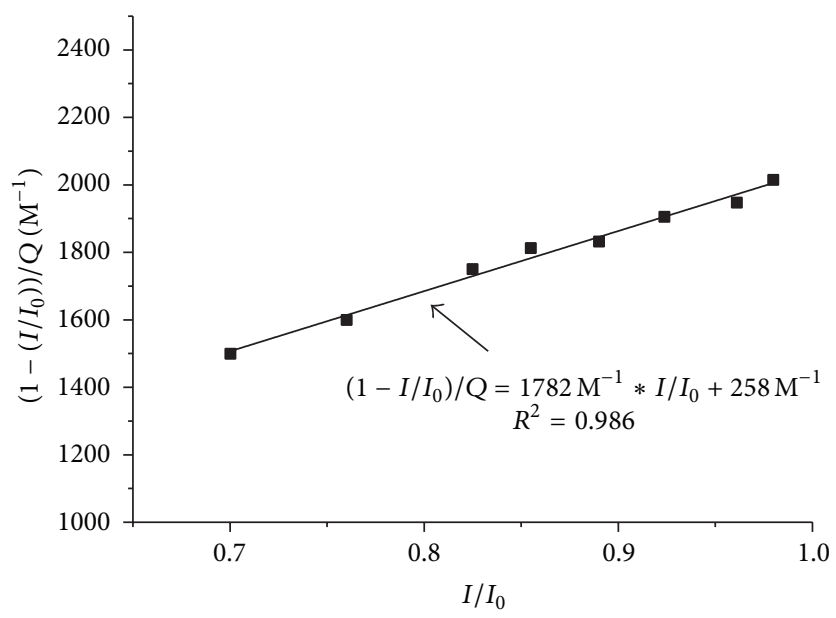

Figure 7: Plot of $\left(1-\left(I / I_{0}\right)\right) / Q$ against $I / I_{0}$ at different concentrations of $\mathbf{1}$.

with the size of molecules, and they correlate well with the values previously reported in the literature for other quenchers. The measured quenching indicates weak molecular level interaction between the anthracene and the cavitand derivative 1; however, it does not prove unambiguously the complex formation.

\section{Conflict of Interests}

The authors declare that there is no conflict of interests regarding the publication of this paper.

\section{Acknowledgments}

The authors gratefully acknowledge the financial support from the Hungarian National Development Agency. Project codes: SROP-4.2.1.B-10/2/KONV-2010-0002, Developing the South-Transdanubian Regional University Competitiveness. SROP-4.2.2.A-11/1/KONV-2012-0065, Synthesis of supramolecular systems, examination of their physicochemical properties and their utilisation for separation and sensor chemistry.

\section{References}

[1] D. J. Cram, S. Karbach, H. Kim et al., "Host-guest complexation. 46. Cavitands as open molecular vessels form solvates," Journal of the American Chemical Society, vol. 110, no. 7, pp. 2229-2237, 1988.

[2] D. J. Cram and J. M. Cram, Container Molecules and Their Guests, Royal Society of Chemistry, Cambridge, UK, 1997.

[3] B. Dietrich, "Comprehensive Supramolecular Chemistry," in Comprehensive Supramolecular Chemistry, G. W. Gokel, Ed., pp. 153-211, Elsevier, Oxford, England, 1996.

[4] C. D. Gutsche and J. F. Stoddart, Calixarenes Revisited, Royal Society of Chemistry, London, UK, 1998.

[5] P. Timmerman, W. Verboom, and D. N. Reinhoudt, "Resorcinarenes," Tetrahedron, vol. 52, no. 8, pp. 2663-2704, 1996.
[6] V. Böhmer, "Calixarenes, macrocycles with (almost) unlimited possibilities," Angewandte Chemie (International Edition), vol. 34, no. 7, pp. 713-745, 1995.

[7] Z. Csók, A. Takátsy, and L. Kollár, "Highly selective palladiumcatalyzed aminocarbonylation and cross-coupling reactions on a cavitand scaffold," Tetrahedron, vol. 68, no. 12, pp. 2657-2661, 2012.

[8] W. Sliwa, "Calixarene- and cavitand-based capsules," Arkivoc, vol. 5, no. 5, pp. 137-159, 2006.

[9] C. Lewis and W. R. Ware, "Wavelength effects in fluorescence quenching: the anthracene-carbon tetrachloride system," Chemical Physics Letters, vol. 15, no. 2, pp. 290-292, 1972.

[10] A. Airinei, R. I. Tigoianu, E. Rusu, and D. O. Dorohoi, "Fluorescence quenching of anthracene by nitroaromatic compounds," Digest Journal of Nanomaterials and Biostructures, vol. 6, no. 3, pp. 1265-1272, 2011.

[11] J. Thipperudrappa, D. S. Biradar, M. T. Lagare, and S. M. Hanagodimath, "Fluorescence quenching of anthracene by aniline in different solvents," Indian Journal of Pure and Applied Physics, vol. 42, no. 9, pp. 648-652, 2004.

[12] P. Kumar Behera and A. Kumar Mishra, "Static and dynamic model for 1-naphthol fluorescence quenching by carbon tetrachloride in dioxane-acetonitrile mixtures," Journal of Photochemistry and Photobiology, A: Chemistry, vol. 71, no. 2, pp. 115118, 1993.

[13] O. Stern and M. Volmer, "Über die abklingzeit der fluoreszenz," Physikalische Zeitschrift, vol. 20, pp. 183-188, 1919.

[14] K. Datta and A. K. Mukherjee, "Study of quenching of anthracene fluorescence by [60]fullerene," Spectrochimica Acta A, vol. 65, no. 2, pp. 261-264, 2006.

[15] F. Galindo, M. I. Burguete, R. Gavara, and S. V. Luis, "Fluorescence quenching in organogel as a reaction medium," Journal of Photochemistry and Photobiology A: Chemistry, vol. 178, no. 1, pp. 57-61, 2006.

[16] T. Arita, O. Kajimoto, M. Terazima, and Y. Kimura, "Experimental verification of the Smoluchowski theory for a bimolecular diffusion-controlled reaction in liquid phase," Journal of Chemical Physics, vol. 120, no. 15, pp. 7071-7074, 2004.

[17] M. Smoluchowski, "Versuch einer mathematischen theorie der koagulationskinetic kolloider lösungen," Zeitschrift für Physikalische Chemie, vol. 92, pp. 129-168, 1917. 
[18] K. Ichikawa, T. Suzuki, and N. Murata, "Stochastic simulation of biological reactions, and its applications for studying actin polymerization," Physical Biology, vol. 7, no. 4, pp. 1-13, 2010.

[19] A. Einstein, "Über die von der molekularkinetischen Theorie der Wärme geforderte Bewegung von in ruhenden Flüssigkeiten suspendierten Teilchen," Annals of Physics, vol. 322, no. 8, pp. 549-560, 1905.

[20] A. Bezrukova, M. Lubomska, and M. Rogalski, "Nanoparticle mixtures of anthracene and $\beta$-cyclodextrin testing by optical spectroscopy," Reviews on Advanced Materials Science, vol. 20, no. 1, pp. 70-76, 2009.

[21] J. R. Lakowicz, Principles of Fluorescence Spectroscopy, Springer, Baltimore, Md, USA, 3rd edition, 2006.

[22] C. A. Parker, "Photoluminescence of solutions," in Photoluminescence of Solutions, vol. 81, pp. 220-226, Elsevier, Amsterdam, The Netherlands, 1968.

[23] M. M. Puchalski, M. J. Morra, and R. von Wandruszka, "Assessment of inner filter effect corrections in fluorimetry," Fresenius' Journal of Analytical Chemistry, vol. 340, no. 6, pp. 341-344, 1991.

[24] H. Zeng and D. Gilles, "Analysis of fluorescence quenching in some antioxidants from non-linear Stern-Volmer plots," Journal of Luminescence, vol. 63, no. 1-2, pp. 75-84, 1995.

[25] H. M. S. Kumar, R. S. Kunabenchi, J. S. Biradar, N. N. Math, J. S. Kadadevarmath, and S. R. Inamdar, "Analysis of fluorescence quenching of new indole derivative by aniline using SternVolmer plots," Journal of Luminescence, vol. 116, no. 1-2, pp. 3542, 2006. 

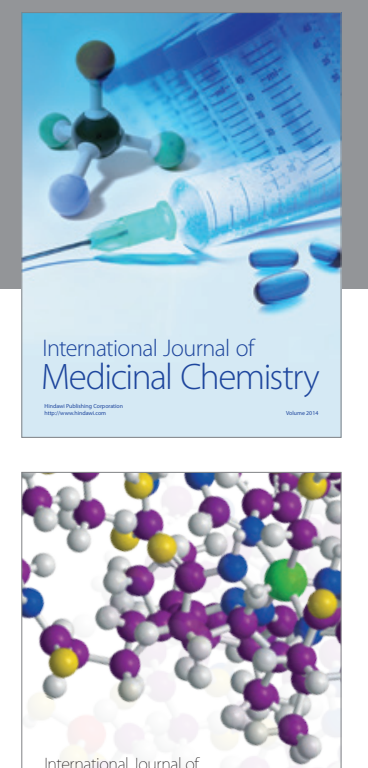

\section{Carbohydrate} Chemistry

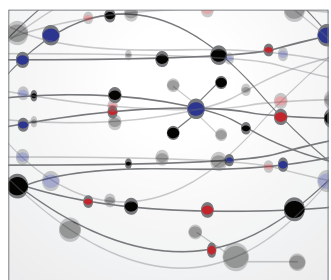

The Scientific World Journal
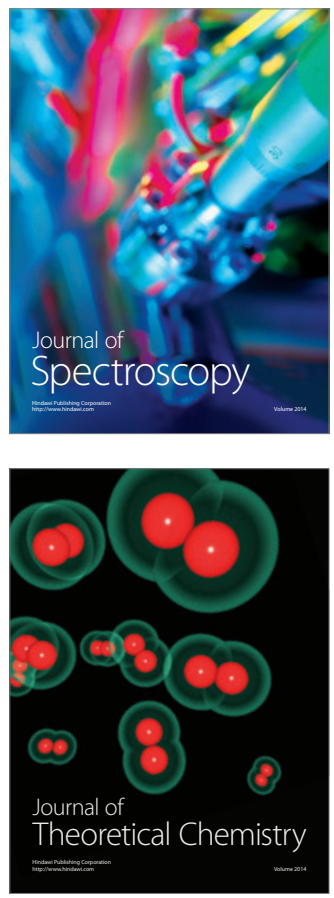
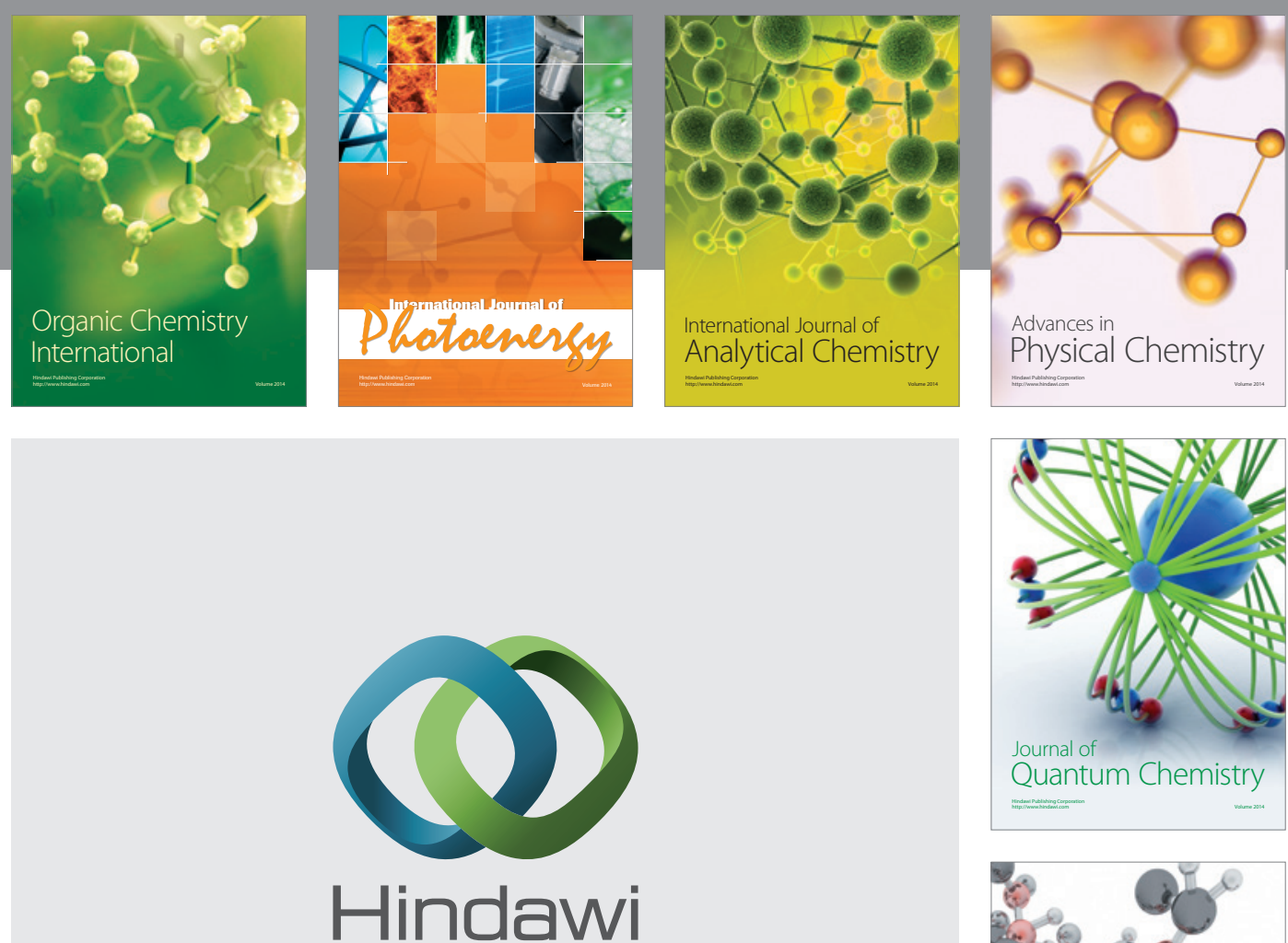

Submit your manuscripts at

http://www.hindawi.com

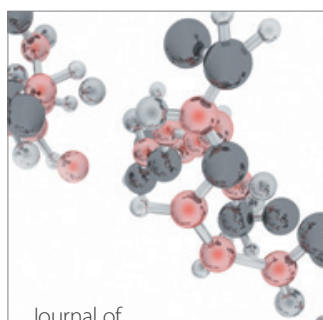

Analytical Methods

in Chemistry

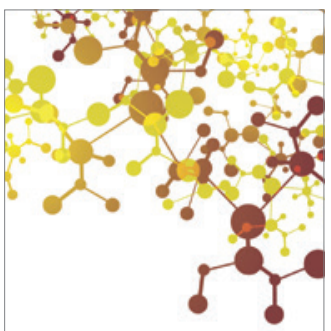

Journal of

Applied Chemistry

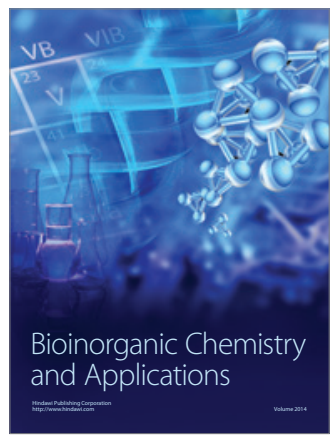

Inorganic Chemistry
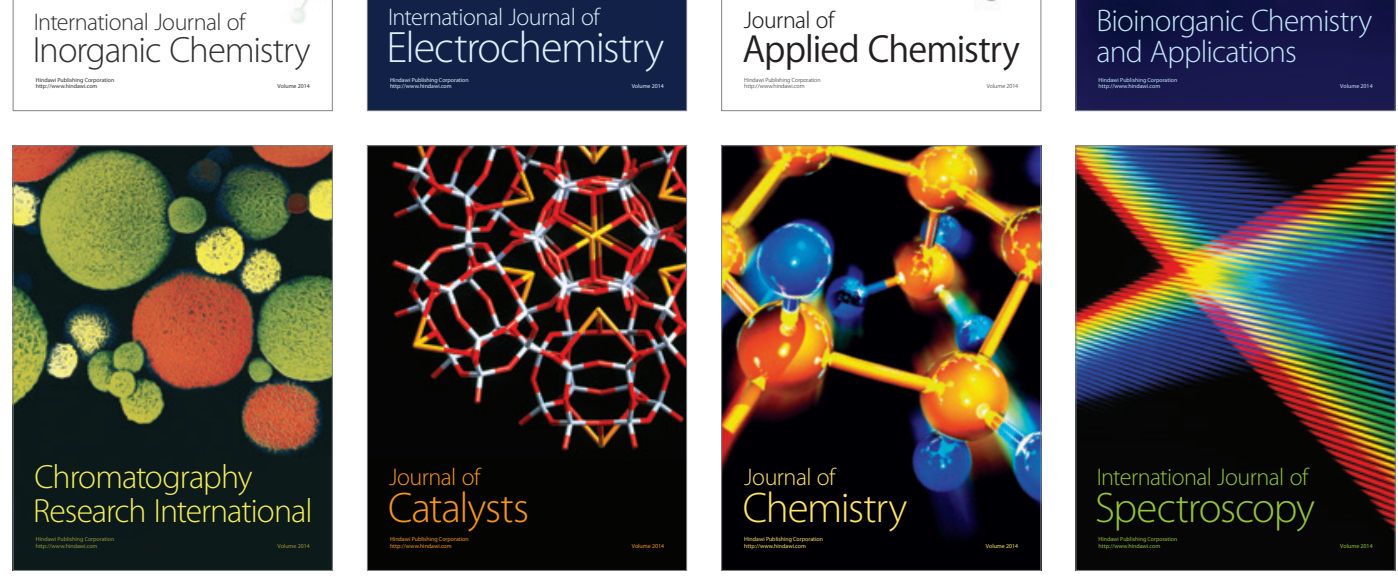